\title{
Low-energy quasi-one-dimensional spin dynamics in charge-ordered $\mathrm{La}_{2-x} \mathrm{Sr}_{x} \mathrm{NiO}_{4}$
}

\author{
P. G. Freeman,,${ }^{1,}$ D. Prabhakaran, ${ }^{2}$ K. Nakajima, ${ }^{3}$ A. Stunault,${ }^{1}$ M. Enderle, ${ }^{1}$ C. Niedermayer, ${ }^{4}$ C. D. Frost,${ }^{5}$ \\ K. Yamada, ${ }^{6}$ and A. T. Boothroyd ${ }^{2}$ \\ ${ }^{1}$ Institut Laue-Langevin, BP 156, F-38042 Grenoble Cedex 9, France \\ ${ }^{2}$ Department of Physics, Oxford University, Oxford OX1 3PU, United Kingdom \\ ${ }^{3}$ Neutron Science Section, MLF Division, J-PARC Center 2-4 Shirane Shirakata, Tokai, Naka, Ibaraki 319-1195, Japan \\ ${ }^{4}$ Laboratory for Neutron Scattering, ETHZ and PSI, CH-5232 Villigen PSI, Switzerland \\ ${ }^{5}$ ISIS Facility, Rutherford Appleton Laboratory, Chilton, Didcot OX11 OQX, United Kingdom \\ ${ }^{6}$ WPI Research Center, Advanced Institute for Materials Research, Tohoku University, Sendai 980-8577, Japan
}

(Received 2 January 2011; published 15 March 2011)

\begin{abstract}
The low-energy spin excitations of $\mathrm{La}_{2-x} \mathrm{Sr}_{x} \mathrm{NiO}_{4}, x=0.275$ and $1 / 3$, have been investigated by unpolarizedand polarized-inelastic neutron scattering from single crystals. A pattern of magnetic diffuse scattering is observed in both compositions and is consistent with quasi-one-dimensional AFM spin correlations along the charge stripes. Analysis of the energy line shape for $x=1 / 3$ indicates that the diffuse scattering is inelastic with a characteristic energy of $1.40 \pm 0.07 \mathrm{meV}$. There is no discernible difference between the diffuse scattering from $x=0.275$ and $x=1 / 3$, suggesting that it is an intrinsic property of the charge stripes.
\end{abstract}

DOI: 10.1103/PhysRevB.83.094414

PACS number(s): 75.40.Gb, 71.45.Lr, 75.30.Fv, 75.30.Et

\section{INTRODUCTION}

Striped patterns of spin and charge order have been observed in a wide range of antiferromagnetic oxides since the initial discovery in $\mathrm{La}_{1.48} \mathrm{Nd}_{0.4} \mathrm{Sr}_{0.12} \mathrm{CuO}_{4} \cdot{ }^{1}$ Interest has been sustained by continuing uncertainty about the true importance of stripe correlations for the mechanism of superconductivity in the layered cuprates. In some models, for example, stripe correlations assist in the formation of pairing instabilities that can lead to superconductivity, ${ }^{2}$ whereas according to experiment static charge stripes suppress superconductivity. ${ }^{1}$ Investigations into the fundamental properties of stripes are therefore potentially important for an understanding of cuprate superconductivity, as well as providing insight into an interesting emergent phase of electronic matter.

Experimental investigations on stripe phases have been made on various materials, but the layered nickelates $\mathrm{La}_{2-x} \mathrm{Sr}_{x} \mathrm{NiO}_{4+\delta}$ (LSNO) have been a particularly informative model system to study. ${ }^{3-8} \mathrm{LSNO}$, which is isostructural with the " 214 " high temperature superconductor $\mathrm{La}_{2-x} \mathrm{Sr}_{x} \mathrm{CuO}_{4+\delta}$, exhibits spin and charge stripe order for $0.15 \leqslant x \leqslant 0.5 .^{9,10}$ The stripes form on the square $\mathrm{NiO}_{2}$ layers and consist of diagonal bands of antiferromagnetically (AFM) ordered $\mathrm{Ni}^{2+}$ spins separated by charged domain walls that act as antiphase boundaries to the magnetic order. At one-third doping (e.g., $x=1 / 3, \delta=0$ ) the stripe order is particularly stable owing to a combination of two factors, first a commensurability effect that pins the charge stripes to the lattice, and second a stripe periodicity which is the same for the magnetic and charge order. ${ }^{9,11,12}$ At this doping level LSNO displays long-range (>100 $\AA$ ) charge order, ${ }^{9,10}$ making it an ideal material in which to probe the charge-ordered state.

The magnetic excitation spectrum in the ordered stripe phase of LSNO with $x \approx 1 / 3, \delta=0$ has been investigated in some detail with neutron inelastic scattering and found to contain two distinct components: (i) quasi-two-dimensional spin-wave excitations of the AFM-ordered regions, extending to $\sim 80 \mathrm{meV}$ in energy, ${ }^{13-16}$ and (ii) a low-energy $(<10 \mathrm{meV})$ quasi-one-dimensional (q-1D) magnetic fluctuation consistent with short-range AFM correlations along the charge stripes. ${ }^{17}$ A two-component spectrum with qualitatively similar characteristics has also been observed in $\mathrm{La}_{3 / 2} \mathrm{Sr}_{1 / 2} \mathrm{NiO}_{4}$ (Ref. 18). The observation of two types of magnetic dynamics is consistent with the existence of two magnetic subsystems associated with nominally $\mathrm{Ni}^{2+}$ and $\mathrm{Ni}^{3+}$ ions, the latter of which form the charge stripes. At this time, however, there is no microscopic model of the magnetic interactions in LSNO that provides a unified description of the complete magnetic spectrum.

The aim of the present study was to work toward a better understanding of the low-energy q-1D magnetic correlations by comparing neutron scattering data from two doping levels, $x=0.275$ and $x=1 / 3$ (both with $\delta=0$ ), and by examining in more detail the energy line shape of the magnetic scattering. We have found that the q-1D scattering is present at both doping levels, and our analysis of the energy line shape reveals that the signal is gapped at the minimum of the q-1D dispersion.

\section{EXPERIMENTAL DETAILS}

Single crystals of $\mathrm{La}_{2-x} \mathrm{Sr}_{x} \mathrm{NiO}_{4}$ were grown by the floating-zone method. ${ }^{19}$ The crystals were in the form of rods with typical dimensions $7-8 \mathrm{~mm}$ in diameter and $\sim 40 \mathrm{~mm}$ in length (mass $\sim 15 \mathrm{~g}$ ).

Neutron scattering measurements were performed on crystals with $x=0.275$ and $x=1 / 3$ on the triple-axis spectrometers (TAS) IN8, IN20, and IN14 at the Institut Laue-Langevin, and on RITA-II at SINQ, Paul Scherrer Institut. The energies of the incident and scattered neutrons were selected by Bragg reflection from crystal arrays of pyrolytic graphite (PG) crystals (IN8, IN14, RITA-II), physically bent Si crystals (IN8), or Heusler arrays (IN20). The monochromators were vertically focused (IN8, IN14) and horizontally focused (IN8, IN20, RITA-II) to maximize neutron flux on the sample position. The analyzers were horizontally focused on all instruments and vertically focused on IN8. Data were collected with fixed 
final neutron wave vectors of $2.662 \AA^{-1}$ (IN8, IN20), $1.5 \AA^{-1}$ (IN14, RITA-II), and $1.2 \AA^{-1}$ (IN14). A pyrolytic graphite filter (IN8, IN20) or Be/BeO filter operating at $77 \mathrm{~K}$ (IN14, RITA-II) was placed between the sample and analyzer to suppress higher order harmonic scattering. On IN20 polarized neutrons were employed, and the neutron spin polarization $\mathbf{P}$ was maintained in a specified orientation with respect to the neutron scattering vector $\mathbf{Q}$ by an adjustable guide field of a few $\mathrm{mT}$ at the sample position. A monitor is placed between the monochromator and sample position to determine the number of neutrons incident on the sample position. The monitor count has an energy dependent contamination due to higher order neutrons in the incident beam, which we have corrected for when integrated intensities are shown. We aligned the crystals so that the horizontal scattering plane was $(h, k, 0)$ for $x=1 / 3$ and $(h, h, l)$ for $x=0.275$ (we refer here to the tetragonal unit cell of the space group $I 4 / \mathrm{mmm}$ with unit cell parameters $a=3.8 \AA$ and $c=12.7 \AA$ ). The particular crystals used in the TAS measurements were grown at Oxford University and have been used in previous neutron scattering studies described elsewhere. ${ }^{13,15,16,21}$

To supplement the TAS measurements we will also present some data on the $x=0.275$ composition collected on the MAPS time-of-flight spectrometer at the ISIS spallation neutron source. A report on the findings of this study at higher energy transfers can be found elsewhere. ${ }^{15}$ The sample used on MAPS was an array of four crystals grown at Kyoto University and co-aligned by $\mathrm{X}$-ray diffraction to within about $1^{\circ}$. Crystals with $x=0.275$ from the same source have been used in neutron diffraction studies of magnetic and charge order. ${ }^{20}$ The sample was mounted on MAPS in a closed-cycle refrigerator and aligned with the $c$ axis parallel to the incident beam direction. A Fermi chopper was used to select the incident neutron energy of $60 \mathrm{meV}$. The intensity was normalized and converted to units of scattering cross section $\left(\mathrm{mb} \mathrm{sr}^{-1} \mathrm{meV}^{-1}\right.$ [f.u. ${ }^{-1}$ ) by comparison with measurements from a standard vanadium sample. Scattered neutrons were recorded in large banks of position-sensitive detectors. The spin dispersion in $\mathrm{La}_{2-x} \mathrm{Sr}_{x} \mathrm{NiO}_{4}$ is highly two-dimensional, ${ }^{15}$ and so we project the data onto the $(h, k)$ two-dimensional reciprocal lattice plane. The elastic energy resolution on MAPS was $2.7 \mathrm{meV}$ (full width at half maximum).

\section{RESULTS}

To help visualise the experimental measurements we reproduce in Fig. 1 part of a figure from our previous publication on the q-1D magnetic fluctuations in $\mathrm{La}_{5 / 3} \mathrm{Sr}_{1 / 3} \mathrm{NiO}_{4}$ (Ref. 17). Figure 1(a) is a simplified map of the low-energy scattering features in the $(h, k, 0)$ plane of reciprocal space. The sharp peaks (circles) at $(1 / 2 \pm 1 / 6,1 / 2 \pm 1 / 6,0)$ and $(1 / 2 \pm$ $1 / 6,1 / 2 \mp 1 / 6,0)$ represent spin-wave scattering associated with the magnetic ordering wave vectors. There are two pairs of peaks because there are two possible orientations of the stripes on the $\mathrm{NiO}_{2}$ layers, either along the $[1, \overline{1}, 0]$ direction or the $[1,1,0]$ direction. The diagonal lines which run parallel to the stripe directions represent the approximate pattern of diffuse scattering observed in $\mathrm{La}_{5 / 3} \mathrm{Sr}_{1 / 3} \mathrm{NiO}_{4}$ (Ref. 17). Figure 1(b) depicts an array of AFM chains running parallel to the stripes which would give rise to the diagonal grid of diffuse scattering

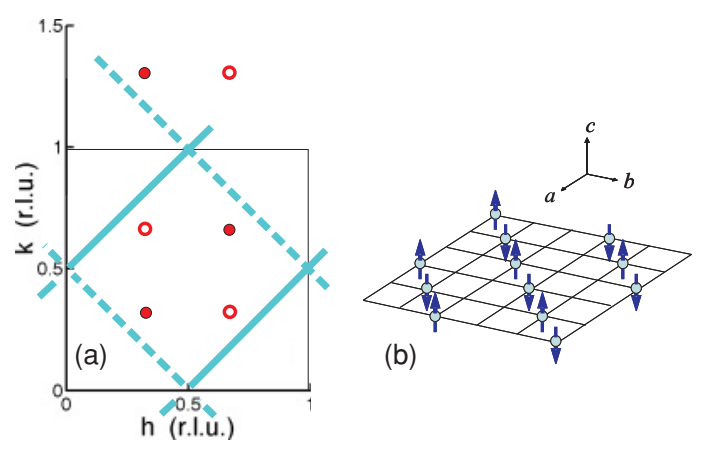

FIG. 1. (Color online) Diagrams representing the low-energy magnetic fluctuations in $\mathrm{La}_{5 / 3} \mathrm{Sr}_{1 / 3} \mathrm{NiO}_{4}$, after Ref. 17. (a) Schematic map of the low-energy scattering in the $(h, k, 0)$ plane of reciprocal space. Circles are stripe superlattice zone centers and diagonal lines indicate the diffuse scattering from ideal one-dimensional AFM spin chains running parallel to the charge stripes. Filled circles and full lines are for stripes running parallel to the $[1, \overline{1}, 0]$ direction; open circles and broken lines are for stripes parallel to the $[1,1,0]$ direction. (b) Model of AFM correlations along the charge stripes consistent with the observed q-1D diffuse scattering. The arrows indicate the instantaneous orientation of the spins on the charge stripes. For clarity, the background AFM order in the regions between the charge stripes is not shown.

shown in Fig. 1(a). In reality, the the line of the observed diffuse scattering is not exactly straight but meanders slightly so as to follow approximately the magnetic zone boundaries, suggesting that the fluctuations on adjacent chains are weakly correlated. The spins are shown pointing upward to reflect the observation that the strength of the out-of-plane fluctuations is about twice the strength of the in-plane fluctuations. ${ }^{17}$ The diffuse scattering has a strong dynamic component on a $\mathrm{THz}$ frequency scale, and may even be entirely dynamic. Whether the q-1D scattering is quasielastic or inelastic (i.e., gapped) is an open question which will be addressed later in this work.

Figure 2 presents maps of the magnetic scattering intensity measured in the $(h, k, 0)$ plane of the $x=1 / 3$ crystal at a temperature of $2 \mathrm{~K}$. The maps were collected at three fixed energies: (a) $0 \mathrm{meV}$ (i.e., elastic scattering within the resolution of the spectrometer), (b) $2.5 \mathrm{meV}$, and (c) $5 \mathrm{meV}$. The $2.5 \mathrm{meV}$ data is reproduced from Ref. 17. Figure 2(d) shows the areas covered in the three maps, which are not the same.

In the elastic map, Fig. 2(a), there is a magnetic Bragg reflection at $(0.667,0.667,0)$ due to the pattern of AFM order between the charge stripes, and a small spurious peak at $(0.45,0.75,0)$. No diffuse elastic scattering signal can be observed within the experimental precision. Comparing the two inelastic maps, Figs. 2(b) and 2(c), one can see that the q-1D diffuse scattering is broader at $E=5 \mathrm{meV}$ than at at $E=2.5 \mathrm{meV}$. This is consistent with our previous measurements ${ }^{17}$ which showed that the q-1D scattering disperses in the direction perpendicular to the scattering ridge, with a bandwidth of about $10 \mathrm{meV}$. The intensity of the q-1D scattering is modulated, with maxima adjacent to the spin-wave scattering from the AFM order and at the positions where the diffuse ridges meet, i.e., $(1,0.5,0)$ and equivalent positions.

We now turn to the LSNO crystal with $x=0.275$. At this composition the charge stripe order is incommensurate with 
$x=1 / 3, k_{f}=2.662 \AA^{-1}:$
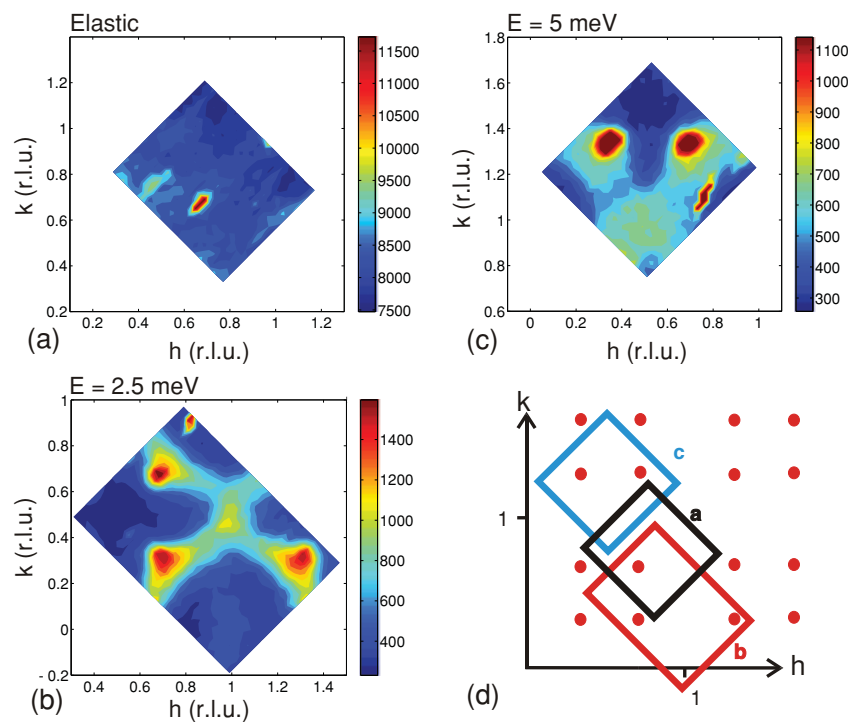

(d)

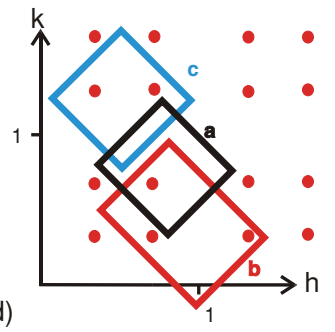

FIG. 2. (Color online) Maps of the scattering intensity in the $(h, k, 0)$ reciprocal space plane of $\operatorname{LSNO}(x=1 / 3)$. (a) Elastic scattering, (b) $E=2.5 \mathrm{meV}$, and (c) $E=5 \mathrm{meV}$. The data were collected on IN8 with a final wave vector of $k_{f}=2.662 \AA^{-1}$ at a temperature of $2 \mathrm{~K}$. The contour plots are formed from $\sim 25 \times 30$ rectangular grids of data points. The data in (a) and (c) were measured with a pyrolytic graphite monochromator, and in (b) with a silicon monochromator. (d) Diagram of reciprocal space indicating the areas mapped out in (a)-(c).

the crystal lattice and it is of interest to see whether this has any effect on the q-1D magnetic fluctuations. Figure 3(a) shows the distribution of scattering intensity from $\operatorname{LSNO}(x=0.275)$ measured on MAPS. The scattering has been averaged over the energy range 3-5 meV and plotted as a function of the in-plane components $(h, k)$ of reciprocal space. There are four strong scattering signals at positions $(0.5 \pm \epsilon / 2,0.5 \pm \epsilon / 2)$ and $(0.5 \pm \epsilon / 2,0.5 \mp \epsilon / 2)$ with $\epsilon=0.297 \pm 0.001$, from the steeply dispersing spin-wave excitations of the AFM order. In Fig. 3(a) the spin-wave excitations from the AFM order are too low in energy to be resolved into spin-wave cones, and appear simply as spots. In addition to these, there can also be seen ridges of diffuse scattering similar to the q-1D scattering observed from the $x=1 / 3$ crystal [Figs. 2(b) and 2(c)]. Figures 3(b) and 3(c) show cuts through this data along the paths marked A and B in Fig. 3(a). Path B is chosen so that no intensity from the excitations of the ordered AFM is observed in a scan along path B. The location for path B is determined experimentally, by performing scans perpendicular to path A through the excitations from the AFM order. The cut in Fig. 3(b) along path A shows the diffuse scattering as a shoulder to the sharper spin-wave peak, whereas the cut shown in Fig. 3(c) along path B shows just the diffuse scattering peak, which is centered on $\xi \approx 0.25$ [the $\xi$ coordinate measures the position along the scan projected perpendicularly onto line A, i.e., such that $\xi=0$ projects onto $(0,0)$ and $\xi=0.5$ projects onto $(0.5,0.5)]$. From these and similar cuts we find that the diffuse scattering at $x=0.275$ follows the same slightly meandering path as does the diffuse scattering at $x=1 / 3$ - see $x=0.275$ :
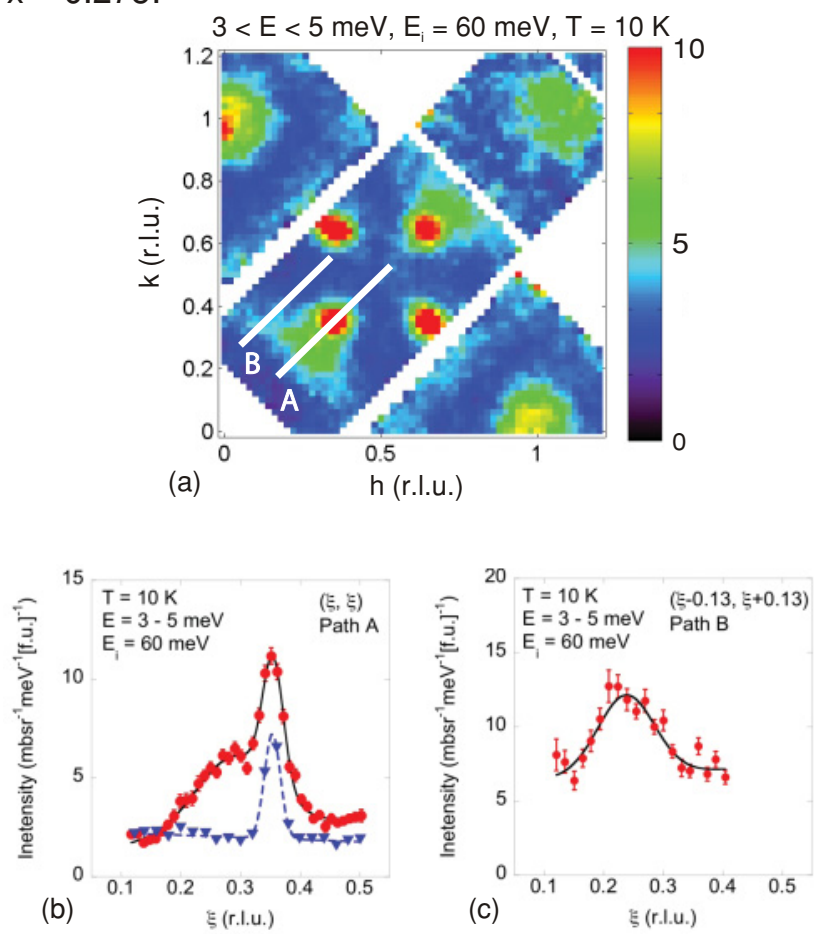

FIG. 3. (Color online) Neutron scattering from $\mathrm{La}_{2-x} \mathrm{Sr}_{x} \mathrm{NiO}_{4}$ $(x=0.275)$ measured on MAPS. (a) Slice through the data volume averaged over the energy range $3-5 \mathrm{meV}$. (b) and (c) Cuts along the paths marked A and B in (a). An elastic scattering cut along path A is also displayed in (b). Solid and dashed lines indicate fits to the data with either one or two Gaussian peaks on a sloping background.

Figs. 2(b) and 2(c). In Fig. 3(b) we also display the elastic scattering intensity along path $\mathrm{A}$. This shows the magnetic Bragg peak from the AFM order but does not contain any elastic signal corresponding to the position of the diffuse inelastic signal.

To confirm that the diffuse scattering signal is magnetic in origin we performed scattering measurements on IN20 employing neutron polarization analysis. For these measurements we constrained the neutron polarization $\mathbf{P}$ to be parallel to the scattering vector $\mathbf{Q}$. In this configuration scattering from electronic magnetic moments causes the neutron spin to flip, whereas scattering via nonmagnetic processes does not.

Figure 4 displays the neutron spin-flip (SF) and nonspin-flip (NSF) scattering from the $x=0.275$ crystal along a line equivalent to path $\mathrm{A}$. Plots of elastic scattering data and inelastic scattering data with $E=3 \mathrm{meV}$ are shown. The elastic scan contains a strong peak in the SF channel centered on $\xi=0.65$, the AFM ordering wave vector for this composition. A small peak in the NSF channel at the same position is due to imperfect spin polarization, which has not been corrected for. At $E=3 \mathrm{meV}$ there is a broad peak centered on $\xi \approx 0.7$ in the SF channel but not in the NSF channel. Due to their very steep dispersion, the spin-wave scattering from the AFM order only accounts for the delta-shaped left side of the SF peak. ${ }^{15}$ The remaining extent of the SF peak can be accounted for by the inelastic diffuse scattering. 


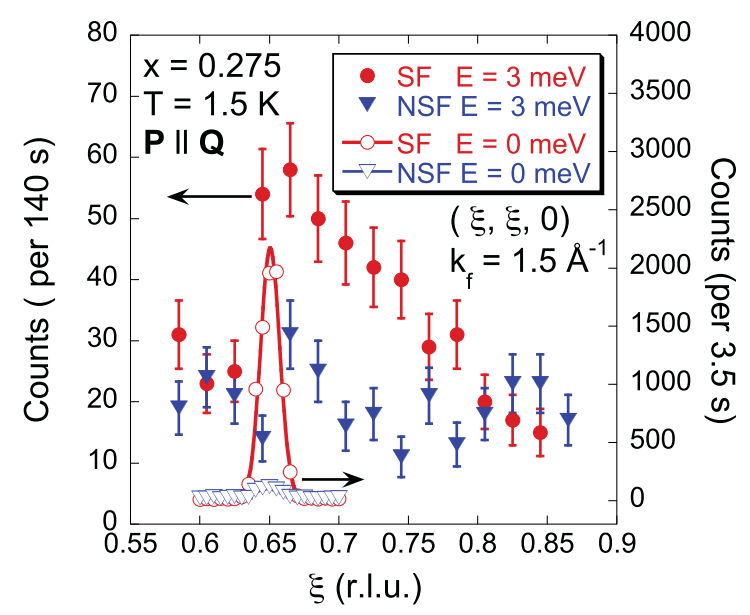

FIG. 4. (Color online) Polarized neutron scattering from $\mathrm{La}_{2-x} \mathrm{Sr}_{x} \mathrm{NiO}_{4}(x=0.275)$. The scans are along the $(1,1,0)$ direction [path A in Fig. 3(a)] and measured at energies of $0 \mathrm{meV}$ (elastic scattering, right scale) and $3 \mathrm{meV}$ (left scale). Spin-flip (SF) and non-spin-flip (NSF) scattering channels are shown. The small peak in the NSF channel of the elastic scan is due to imperfect polarization, which has not been corrected for.

The results of the unpolarized and polarized neutron scattering measurements presented here for $x=0.275$ are qualitatively very similar to our previous measurements ${ }^{17}$ on $x=1 / 3$. This shows that the $\mathrm{q}-1 \mathrm{D}$ diffuse scattering is magnetic in origin and has the same character in both the $x=0.275$ and $x=1 / 3$ samples.

Next we turn to the question of whether the q-1D scattering is gapped or not. To answer this we performed inelastic neutron scattering measurements on IN14 on the $x=1 / 3$ crystal at energies $E \leqslant 3 \mathrm{meV}$. Figure 5 shows constant-energy scans at $0.2 \mathrm{meV}$ and $0.8 \mathrm{meV}$ along a direction equivalent to path $\mathrm{B}$ of Fig. 1(a). The scan at $0.8 \mathrm{meV}$ reveals a peak centered on $\xi=$ 0.75 from the q-1D fluctuation, whereas the scan at $0.2 \mathrm{meV}$ shows no peak at $\xi \sim 0.75$ within the statistical precision of the data. The peak widths of both the q-1D diffuse scattering at $0.8 \mathrm{meV}$ and the spin-wave scattering at $0.6 \mathrm{meV}$ (not shown) are resolution limited. ${ }^{22}$

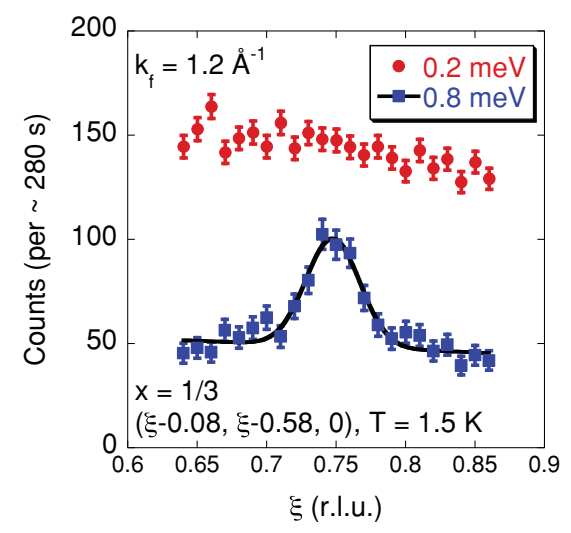

FIG. 5. (Color online) Constant-energy scans along path B for the $\mathrm{La}_{2-x} \mathrm{Sr}_{x} \mathrm{NiO}_{4}(x=1 / 3)$ at $0.2 \mathrm{meV}$ and $0.8 \mathrm{meV}$. The solid line is the best fit to a Gaussian function on a linear background.

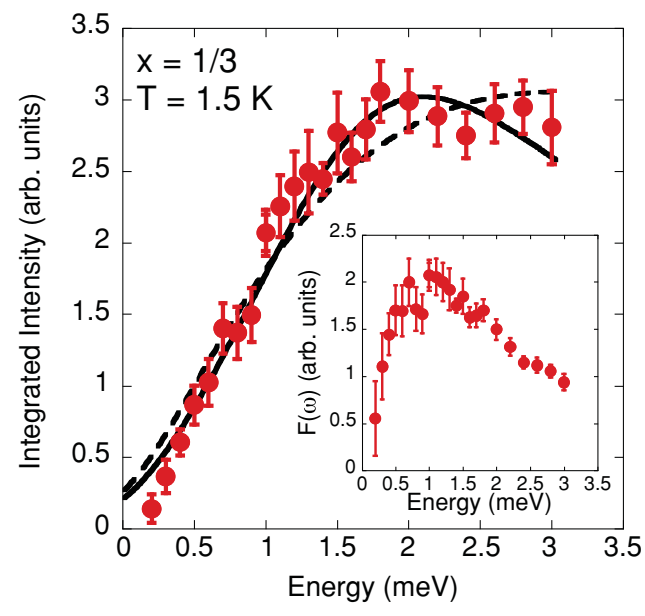

FIG. 6. (Color online) Line shape of the q-1D diffuse scattering in $\mathrm{La}_{2-x} \mathrm{Sr}_{x} \mathrm{NiO}_{4}(x=1 / 3)$ at low energy. The integrated intensities were obtained from the area of a Gaussian function fitted to a series of constant-energy scans like those shown in Fig. 5. For $E \leqslant 0.6$ $\mathrm{meV}$ the signal was small and the Gaussian width was fixed in the fit to the value obtained from the $0.8 \mathrm{meV}$ data. The measurements were made with $k_{f}=1.2 \AA^{-1}$ for $E \leqslant 1 \mathrm{meV}$ and $k_{f}=1.5 \AA^{-1}$ for $E \geqslant 1 \mathrm{meV}$, and normalized so as to match at $1 \mathrm{meV}$. The solid line is calculated from Eq. (1) with $E_{0}=1.4 \mathrm{meV}$, and the broken line is calculated with $E_{0}=0$. The inset shows the spectral weight function, which is related to the dynamical susceptibility by $\chi^{\prime \prime}(\omega) \propto \omega F(\omega)$.

By performing a series of constant-energy scans like those of Fig. 5 and fitting these with Gaussian peaks we determined the integrated intensity of the spin-wave and q-1D diffuse scattering peaks as a function of energy. We remind the reader that in scans along path $\mathrm{B}$ only the q-1D excitations are observed, while in scans along path A the excitations from the AFM order are clearly resolved from the q-1D. ${ }^{17}$ Below $3 \mathrm{meV}$ we observed no energy variation of the integrated intensity of the spin excitations from the AFM order along path $\mathrm{A}$ (not shown), placing an upper limit of $0.3 \mathrm{meV}$ on the in-plane anisotropy gap. In Fig. 6 the energy variation of the integrated intensity of the diffuse scattering peak in $x=1 / 3$ is plotted. With increasing energy transfer the integrated intensity of the diffuse scattering increases monotonically up to $\sim 2$ $\mathrm{meV}$, then remains almost constant up to $3 \mathrm{meV}$, the highest energy measured.

In a separate experiment we investigated the q-1D diffuse scattering from the $x=0.275$ sample, performed on the RITAII spectrometer. In the inset of Fig. 7 we show constant-energy scans at $0.8 \mathrm{meV}$ and $0.5 \mathrm{meV}$ along a direction equivalent to path A. For $E=0.8 \mathrm{meV}$ the q-1D scattering is clearly observed and centered on $\xi=0.73$. At $E=0.5 \mathrm{meV}$ there is still a small excess of scattering above the background at $\xi=$ 0.73 . The centering is consistent with the meandering of the q1D observed in Fig. 2, and the energy dependence is consistent with our observations of the $\mathrm{q}-1 \mathrm{D}$ in the $x=1 / 3$ sample; see Fig. 5 and Fig. 6. Figure 7 shows a constant-Q scan at $\mathbf{Q}=(0.75,0.75,0)$ of the background-corrected amplitude of the q-1D in the $x=0.275$, off-centered to avoid the excitations from the AFM order. The background was estimated from measurements at nearby wave vectors. With decreasing energy 


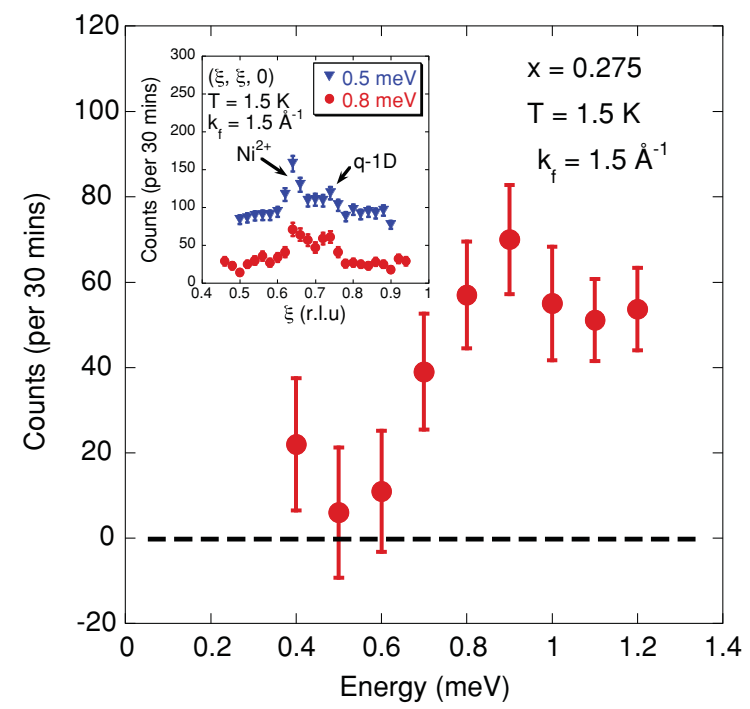

FIG. 7. (Color online) Background-corrected energy scan of the q-1D diffuse scattering from $\mathrm{La}_{2-x} \mathrm{Sr}_{x} \mathrm{NiO}_{4}(x=0.275)$ at $\mathbf{Q}=$ $(0.75,0.75,0)$. The background was estimated from energy scans at $(0.75,0.75,0) \pm(0.19,0.19,0)$. Inset: Constant-energy scans along path $\mathrm{A}$ at energies of $0.5 \mathrm{meV}$ and $0.8 \mathrm{meV}$. Arrows indicate the positions of the spin-wave scattering from the AFM order of the $\mathrm{Ni}^{2+}$ spins and from the q-1D diffuse scattering. The $0.5 \mathrm{meV}$ scan has been offset by the addition of 50 counts for clarity.

transfer the signal remains roughly constant down to $0.8 \mathrm{meV}$, then drops to a level close to zero at around $0.5 \mathrm{meV}$. Within the limitations of low counting statistics, this is consistent with the constant energy scans on the $x=0.275$ sample, and our observations of the $\mathrm{q}-1 \mathrm{D}$ in the $x=1 / 3$ sample; see Fig. 6.

\section{DISCUSSION}

This work has been concerned with the nature of the low-energy q-1D diffuse scattering in $\mathrm{La}_{2-x} \mathrm{Sr}_{x} \mathrm{NiO}_{4}$ first found for $x=1 / 3$ (Ref. 17). We have shown here that the diffuse scattering is also present at $x=0.275$, and that it is the same for $x=0.275$ as for $x=1 / 3$ to within the experimental precision. This implies that the q-1D scattering is not dependent on the periodicity of the spin-charge stripe order, and neither is it a consequence of the special circumstance found at $x=1 / 3$ in which the spin and charge order have the same periodicity and are commensurate with the crystal lattice. Instead, the diffuse scattering appears to be an intrinsic property of individual charge stripes embedded in the AFM matrix formed by the $\mathrm{Ni}^{2+}$ spins, consistent with the interpretation in terms of q-1D correlations among spins in the charge stripes. ${ }^{17}$

In this work we have also carefully measured the lowenergy line shape of the q-1D diffuse scattering. To model the line shape we recall that the dynamical part of the scattering is proportional to the linear response function

$$
S(\mathbf{Q}, \omega)=\frac{1}{\pi}\{n(\omega)+1\} \chi^{\prime \prime}(\mathbf{Q}, \omega),
$$

where $\hbar \omega$ is the energy transferred to the system, $\chi^{\prime \prime}(\mathbf{Q}, \omega)$ is the imaginary part of the dynamical susceptibility, and

$$
n(\omega)=\frac{1}{\exp (\beta \hbar \omega)-1} .
$$

To describe the Q-integrated intensities we used the phenomenological Lorentzian line shape constructed to satisfy detailed balance,

$$
\chi^{\prime \prime}(\omega)=\left[\frac{\Gamma \omega / \pi}{\left(\hbar \omega-E_{0}\right)^{2}+\Gamma^{2}}+\frac{\Gamma \omega / \pi}{\left(\hbar \omega+E_{0}\right)^{2}+\Gamma^{2}}\right],
$$

where $\Gamma$ is the Lorentzian width (half-width at half maximum) and $E_{0}$ is the energy of the undamped mode. As the q-1D has a large intrinsic energy width, $E_{0}$ is the characteristic energy for the q-1D, not a gap energy.

The best fit to the data achieved by this line shape is shown as the solid line in Fig. 6, and gives an energy $E_{0}=1.40 \pm$ $0.07 \mathrm{meV}$ and $\Gamma=1.57 \pm 0.13 \mathrm{meV}$. The fact that the fitted $E_{0}$ is nonzero provides a clear indication that the q-1D diffuse scattering is inelastic rather than quasielastic. To assess how robust this result is we repeated the fit with $E_{0}$ fixed at zero, corresponding to a quasielastic line shape. The best fit thus obtained is shown with a broken line in Fig. 6. The width of the quasielastic fit was $\Gamma=3.0 \pm 0.3 \mathrm{meV}$. The fit to the inelastic line shape has a goodness-of-fit parameter $\chi^{2}=1.19$ compared with a $\chi^{2}=2.25$ for the quasielastic line shape. On the strength of this evidence we conclude that q-1D diffuse scattering corresponds to a gapped inelastic excitation.

For an alternative representation of the inelastic line shape we show in the inset to Fig. 6 the spectral weight function $F(\omega)$, which is related to the imaginary part of the dynamical susceptibility by $\chi^{\prime \prime}(\omega) \propto \omega F(\omega)$. The large energy width of the excitations indicates that the q-1D excitations are relatively short lived, $\Delta t \sim \hbar / \Gamma \sim 4 \times 10^{-13} \mathrm{~s}$.

In our original work on the q-1D spin correlations in LSNO with $x=1 / 3$ we analyzed the magnetic spectrum with respect to that of an antiferromagnetic spin chain. ${ }^{17}$ Assuming the doped holes reside in localized $\mathrm{Ni}^{3+}$ states with low-spin $S=$ $1 / 2$ this implies that the fundamental excitations are spinons with a gapless dispersion. In the present study, however, we have presented evidence that the spectrum has a gap with a characteristic energy of $1.4 \mathrm{meV}$. Further thought is needed, therefore, if we are to reconcile this information with our understanding of the q-1D magnetic diffuse scattering.

The model of an AFM spin chain to describe the spin correlations along the $\mathrm{Ni}^{3+}$ charge stripes is reasonable, provided that the coupling between the spin chain and the AFM order of the $\mathrm{Ni}^{2+}$ spins can be neglected. The justification for neglect of this coupling is that the net Heisenberg exchange acting on the $\mathrm{Ni}^{3+}$ sites from the AFM-ordered $\mathrm{Ni}^{2+}$ spins cancels at the mean-field level, so the coupling between the spin chain and the AFM order is frustrated. However, if the individual $\mathrm{Ni}^{3+}-\mathrm{Ni}^{2+}$ exchange interactions are strong enough then a weak ferromagnetic (FM) order could be induced on the $\mathrm{Ni}^{3+}$ stripes combined with a canting of the AFM order of the $\mathrm{Ni}^{2+}$ spins. Such a possibility has been proposed by Klingeler et al. based on magnetization data. ${ }^{24}$ Weak FM order would be difficult to detect in diffraction experiments because the associated magnetic Bragg peaks would coincide with the Bragg peaks from the crystal lattice. Nevertheless, the competition 
between induced FM order and AFM correlations from an effective AFM exchange along the stripes could provide an explanation for the observed gapped dispersion. Such a model has been investigated recently with promising results. ${ }^{25}$

\section{CONCLUSION}

This work has revealed that gapped, quasi-1D AFM spin correlations are an intrinsic property of the charge stripes in $\mathrm{La}_{2-x} \mathrm{Sr}_{x} \mathrm{NiO}_{4}$. The findings should inform theoretical models for the magnetic interactions in LSNO, and hence contribute to a broader understanding of the formation and stability of spin-charge stripes in LSNO and related systems.

\section{ACKNOWLEDGMENTS}

The authors would like to acknowledge the help of H. J. Woo in the MAPS experiment. This work was performed in part at the Swiss Spallation Neutron Source SINQ, at the Paul Scherrer Institute (PSI), Villigen, Switzerland. We are grateful for support from the Engineering and Physical Sciences Research Council of Great Britain, the European Commission under the 7th Framework Programme through the "Research Infrastructures" action of the "Capacities" Programme, Contract No. CP-CSA INFRA-2008-1.1.1 Number 226507-NMI3, and the support of a Grant-in-Aid for Scientific Research (No. 22244039) from the MEXT, Japan. *freeman@ill.fr

${ }^{1}$ J. M. Tranquada, B. J. Sternleib, J. D. Axe, Y. Nakamura, and S. Uchida, Nature (London) 375, 561 (1995).

${ }^{2}$ H. J. Schulz, J. Phys. (France) 50, 2833 (1989); Phys. Rev. Lett. 64, 1445 (1990); D. Poilblanc and T. M. Rice, Phys. Rev. B 39, 9749 (1989); J. Zaanen and O. Gunnarsson, ibid. 40, 7391 (1989); K. Machida, Physica C 158, 192 (1989); M. Kato, K. Machida, H. Nakanishi, and M. Fujita, J. Phys. Soc. Jpn., 59, 1047 (1990); V. J. Emery and S. A. Kivelson, Physica C 209, 597 (1993); H. Johannesson and G. I. Japaridze, Phys. Rev. B 68, 214507 (2003). ${ }^{3}$ S. M. Hayden, G. H. Lander, J. Zarestky, P. J. Brown, C. Stassis, P. Metcalf, and J. M. Honig, Phys. Rev. Lett. 68, 1061 (1992); V. Sachan, D. J. Buttrey, J. M. Tranquada, J. E. Lorenzo, and G. Shirane, Phys. Rev. B 51, 12742 (1995); J. M. Tranquada, D. J. Buttrey, and V. Sachan, ibid. 54, 12318 (1996).

${ }^{4}$ E. D. Isaacs, G. Aeppli, P. Zschack, S-W. Cheong, H. Williams, and D. J. Buttrey, Phys. Rev. Lett. 72, 3421 (1994); A. Vigliante, M. von Zimmermann, J. R. Schneider, T. Frello, N. H. Andersen, J. Madsen, D. J. Buttrey, Doon Gibbs, and J. M. Tranquada, Phys. Rev. B 56, 8248 (1997).

${ }^{5}$ C. H. Chen, S-W. Cheong, and A. S. Cooper, Phys. Rev. Lett. 71, 2461 (1993).

${ }^{6}$ K. Yamada, T. Omata, K. Nakajima, Y. Endoh, and S. Hosoya, Physica C 221, 355 (1994).

${ }^{7}$ Yu. G. Pashkevich, V. A. Blinkin, V. P. Gnezdilov, V. V. Tsapenko, V. V. Eremenko, P. Lemmens, M. Fischer, M. Grove, G. Güntherodt, L. Degiorgi, P. Wachter, J. M. Tranquada, and D. J. Buttrey, Phys. Rev. Lett. 84, 3919 (2000).

${ }^{8}$ Y. Yoshinari, P. C. Hammel, and S.-W. Cheong, Phys. Rev. Lett. 82, 3536 (1999).

${ }^{9}$ H. Yoshizawa, T. Kakeshita, R. Kajimoto, T. Tanabe, T. Katsufuji, and Y. Tokura, Phys. Rev. B 61, R854 (2000); Physica B 241-243, 880 (1998).

${ }^{10}$ P. D. Hatton, M. E. Ghazi, S. B. Wilkins, P. D. Spencer, D. Mannix, T. d'Almeida, D. Prabhakaran, A. T. Boothroyd, and S.-W. Cheong, Physica B 318, 289 (2002).

${ }^{11}$ A. P. Ramirez, P. L. Gammel, S-W. Cheong, D. J. Bishop, and P. Chandra, Phys. Rev. Lett. 76, 447 (1996).
${ }^{12}$ R. Kajimoto, T. Kakeshita, H. Yoshizawa, T. Tanabe, T. Katsufuji, and Y. Tokura, Phys. Rev. B 64, 144432 (2001).

${ }^{13}$ A. T. Boothroyd, D. Prabhakaran, P. G. Freeman, S. J. S. Lister, M. Enderle, A. Hiess, and J. Kulda, Phys. Rev. B 67, 100407(R) (2003).

${ }^{14}$ P. Bourges, Y. Sidis, M. Braden, K. Nakajima, and J. M. Tranquada, Phys. Rev. Lett. 90, 147202 (2003).

${ }^{15}$ H. Woo, A. T. Boothroyd, K. Nakajima, T. G. Perring, C. D. Frost, P. G. Freeman, D. Prabhakaran, K. Yamada, and J. M. Tranquada, Phys. Rev. B 72, 064437 (2005).

${ }^{16}$ A. T. Boothroyd, P. G. Freeman, D. Prabhakaran, M. Enderle, and J. Kulda, Physica B 345, 1 (2004).

${ }^{17}$ A. T. Boothroyd, P. G. Freeman, D. Prabhakaran, A. Hiess, M. Enderle, J. Kulda, and F. Altorfer, Phys. Rev. Lett. 91, 257201 (2003).

${ }^{18}$ P. G. Freeman, A. T. Boothroyd, D. Prabhakaran, C. D. Frost, M. Enderle, and A. Heiss, Phys. Rev. B 71, 174412 (2005).

${ }^{19}$ D. Prabhakaran, P. Isla, and A. T. Boothroyd, J. Cryst. Growth 237-239, 815 (2002).

${ }^{20}$ S-H. Lee, S.-W. Cheong, K. Yamada, and C. F. Majkrzak, Phys. Rev. B 63, 060405(R) (2001).

${ }^{21}$ P. G. Freeman, A. T. Boothroyd, D. Prabhakaran, M. Enderle, and C. Niedermayer, Phys. Rev. B 70, 024413 (2004); P. G. Freeman, N. B. Christensen, D. Prabhakaran, and A. T. Boothroyd, J. Phys. Conf. Ser. 200, 012037 (2010).

${ }^{22}$ The resolution functions for IN14 were calculated using the RESTRAX program. ${ }^{23}$ For $k_{f}=1.2 \AA^{-1}$ the resolution ellipsoid was typically of dimensions $0.06 \mathrm{meV}$ in energy and $0.025 \AA^{-1}$ parallel and perpendicular to the scan directions. For $k_{f}=1.5 \AA^{-1}$ the resolution ellipsoid was typically of dimensions $0.14 \mathrm{meV}$ in energy, and $0.025 \AA^{-1}$ and $0.035 \AA^{-1}$ parallel and perpendicular to the scan direction, respectively.

${ }^{23}$ J. Šaroun and J. Kulda, Physica B 234-236, 1102 (1997).

${ }^{24}$ R. Klingeler, B. Büchner, S.-W. Cheong, and M. Hücker, Phys. Rev. B 72, 104424 (2005).

${ }^{25}$ M. W. Long and M. Hopper (unpublished). 